\title{
Estimation of Conditional Weighted Expected Shortfall under Adjusted Extreme Quantile Autoregression
}

\author{
Martin M. Kithinji ${ }^{*}{ }^{\oplus}$, Peter N. Mwita² ${ }^{(0)}$, Ananda 0. Kube ${ }^{3}$ \\ ${ }^{1}$ Department of Mathematics, Institute for Basic Sciences, Technology and Innovation, Pan African University, Nairobi, Kenya \\ ${ }^{2}$ Department of Mathematics, Machakos University, Machakos, Kenya \\ ${ }^{3}$ Department of Statistics and Actuarial Sciences, Kenyatta University, Nairobi, Kenya \\ Email: ^mkithinji@kyu.ac.ke
}

How to cite this paper: Kithinji, M.M., Mwita, P.N. and Kube, A.O. (2021) Estimation of Conditional Weighted Expected Shortfall under Adjusted Extreme Quantile Autoregression. Journal of Mathematical Finance, 11, 373-385.

https://doi.org/10.4236/jmf.2021.113021

Received: April 20, 2021

Accepted: July 11, 2021

Published: July 14, 2021

Copyright $\odot 2021$ by author(s) and Scientific Research Publishing Inc. This work is licensed under the Creative Commons Attribution International License (CC BY 4.0).

http://creativecommons.org/licenses/by/4.0/

\begin{abstract}
In this paper, we present an estimator that improves the well-calibrated coherent risk measure: expected shortfall by restructuring its functional form to incorporate dynamic weights on extreme conditional quantiles used in its definition. Adjusted Extreme Quantile Autoregression will is used in estimating intermediary location measures. Consistency and coherence of the estimator are also proved. The resulting estimator was found to be less conservative compared to the expected shortfall.
\end{abstract}

\section{Keywords}

Exreme Quantile Autoregression, Expected Shortfall, Value at Risk, Coherence, Risk Measurement

\section{Introduction}

Different regulators expect institutions under their jurisdiction to comply with the set guidelines on how to ensure capital adequacy. A major threat to capital adequacy is reserves for fluctuations in investment values due to market risk. The amount of this reserve is determined using risk measures that quantify the market risk from the downside distribution of returns. One of the basic risk measures is volatility commonly referred to as standard deviation. Popular measures of risk that have found immense use in financial risk management include Value at Risk (VaR) and Expected Shortfall (ES).

$\mathrm{VaR}$ is the maximum potential loss a portfolio can suffer at a certain confidence interval, say $99 \%$, in a particular number of days called the holding period. 
In addition to comparing Value at Risk and the coherent Expected Shortfall proposed in [1], it was also noted in [2] that VaR improved the "what if?" sensitivity analysis in Greeks by expressing risk in terms of currency as one number with some probability attached. This eases risk reporting and aids in comparing different portfolios. However, VaR suffers from two major shortcomings that cripple its application in risk measurement. These are: $\mathrm{VaR}$ is not sub-additive with respect to subportfolios as well as risk variables and the fact that it underestimates true risk by failing to appreciate the severity of losses beyond the confidence threshold, [3]. Moreover, [4] showed that it is possible to construct two portfolios with different levels of tail risk but with the same VaR.

To remedy the above shortcomings the Basel Committee on Banking Supervision during the fundamental review of the trading book in the year 2013 adopted 97.5\% Expected shortfall with a horizon of one day in place of $99 \% \mathrm{VaR}$ in quantifying market risk for banks under the Basel III framework, [5]. This decision was followed by a lot of criticism based on earlier findings in [6] that ES is not elicitable and hence impossible to backtest. The fears were quelled by [7] who derived backtest procedures for ES that do not require elicitability. In fact elicitability makes backtesting easier but is not a necessary condition for backtesting procedures, [4]. However, following findings in [8] that ES is less robust compared to $\mathrm{VaR}$, any misspecification of the loss/returns distribution greatly affects the risk estimates from ES. This can be attributed to the fact that ES averages quantiles in the extreme left tail of the returns distribution (right tail of the loss distribution) with equal weights meaning that the very extreme quantiles have the same effect on the final estimate as quantiles close to VaR.

The use of equal weights was remedied in [9] using a uniformly weighted sum of a systematic sample of quantiles below the value at risk as an estimator of the expected shortfall. The number of sample quantiles used in the estimation was chosen subjectively depending on the considered sample size of returns distribution. This approach was generalized in [10] through the use of nonuniform weights in the summation of the sample quantiles below the value at risk; where the left tail of the returns distribution was considered. The weights were defined using a continuously differentiable function that assigned more weight to quantiles near $\alpha$ thus improving the asymptotic efficiency of the ES estimator.

This paper, presents derivation of an estimator for Weighted Expected Shortfall (WES) based on extreme quantile regression in [11] to remedy the subjectivity in the WICQF ${ }^{1}$ estimator in [10]. To achieve this, dynamic weights are introduced in the structural form of expected shortfall. The weights depend on the distance between the extreme quantiles and the value at risk. This will allow us to use all the quantiles above VaR thus quashing the subjectivity in WICQF estimator.

\section{Methodology}

Consider a real valued financial time series $S_{t}, t \in \mathbb{R}^{+} \cup\{0\}$ on a complete prob${ }^{1}$ WICQF, Weighted Conditional Integrated Quantile Function. 
ability space $(\Omega, \mathcal{F}, P)$. Assume $S_{t}$ is $\mathcal{F}_{t}$-measurable where $\left\{\mathcal{F}_{t}, t \in \mathbb{R}^{+} \cup\{0\}\right\}$ is an increasing sequence of $\sigma$-algebras representing information available up to time $t$. In particular, let $S_{t}$ be the value of a portfolio at trading time $t$ so that the return, $r_{t}$ on the portfolio at time $t$ is given by

$$
r_{t}=\frac{S_{t}-S_{t-\Delta t}}{S_{t-\Delta t}}
$$

For convenience, let the corresponding loss return be given by

$$
X_{t}=-r_{t}
$$

The $\mathrm{VaR}$ at $(1-\alpha) \times 100 \%$ of the return on this portfolio is given by the quantile at $\alpha \times 100 \%$ of the loss distribution

$$
q_{\alpha}^{x}=\inf \{x \in \mathbb{R}: P(X>x) \leq 1-\alpha\}=\inf \left\{x \in \mathbb{R}: F_{X}(x)>\alpha\right\}
$$

where $\alpha \in(0,1)$. The corresponding ES of the return on this portfolio is the expected value of the losses beyond $\mathrm{VaR}$ which is given by

$$
E S_{\alpha}^{x}=E\left[X \mid X>q_{\alpha}^{x}\right]=\frac{1}{1-\alpha} \int_{\alpha}^{1} q_{\tau}^{x} \mathrm{~d} \tau=q_{\alpha}^{x}+\frac{1}{1-\alpha} E\left[X-q_{\alpha}^{X} \mid X>q_{\alpha}^{X}\right]
$$

where $q_{\alpha}^{X}$ is the inverse of the loss distribution function $F_{X}(x)$

For a discrete ordered sample $x_{1} \leq x_{2} \leq \cdots \leq x_{n}$ the corresponding VaR and ES estimates are respectively given by

$$
\begin{gathered}
\hat{q}_{\alpha}^{x}=x_{n \alpha} \\
\widehat{E S}_{\alpha}^{x}=\frac{1}{n-n \alpha} \sum_{i=n \alpha}^{n} x_{i}
\end{gathered}
$$

where appropriate approximations are made when $n \alpha$ is not an integer.

Let us now define a conditional quantile autoregressive model on $X_{t}$ of the form

$$
X_{t}=\mu_{t, \theta}+\varepsilon_{t}
$$

where $\mu_{t, \theta} \equiv f: \mathbb{R}^{d} \rightarrow \mathbb{R}$ is the central conditional $\theta$-quantile of $X_{t}$ and $\varepsilon_{t}$ are heteroscedastic errors with zero $\theta$-quantile. Suppose we define $\varepsilon_{t}=\sigma_{t, \theta} Z_{t}$ where $\sigma_{t, \theta} \equiv f: \mathbb{R}^{d} \rightarrow \mathbb{R}$ is the central conditional scale of $X_{t}$ and $Z_{t}$ are assumed to be $\mathrm{iid}^{2}$ innovations with a common distribution $F($.). To capture the ARCH effects in $X_{t}$ we let $\sigma_{t, \theta}$ be a function of lagged values of $\varepsilon_{t}$, that is $\left|\varepsilon_{t-i}\right| ; i \in \mathbb{N}$. This modification improves the QAR-QAR process in [11]

According to [11] the adjusted extreme conditional quantile of $X_{t}$ is given by

$$
\mu_{t, \theta, \alpha}=\mu_{t, \theta}+\sigma_{t, \theta}\left[q_{\alpha}^{z}-q_{\theta}^{z}\right]
$$

defined such that if estimates of the quantiles of $Z_{t}$ at $\alpha$ and $\theta$ are $\hat{q}_{\alpha}^{z}$ and $\hat{q}_{\theta}^{z}$ respectively then we can estimate the function by

$$
\hat{\mu}_{t, \theta, \alpha}=\hat{\mu}_{t, \theta}+\hat{\sigma}_{t, \theta}\left[\hat{q}_{\alpha}^{z}-\hat{q}_{\theta}^{z}\right]
$$

${ }^{2}$ Independent and identically distributed. 


\section{Estimation of Common Risk Measures}

Using Equation (9) we obtain an estimator for the one step ahead VaR forecast as

$$
\widehat{\operatorname{VaR}}_{t+1}^{\alpha}=\hat{\mu}_{t+1, \theta, \alpha}=\hat{\mu}_{t+1, \theta}+\hat{\sigma}_{t+1, \theta}\left[\hat{q}_{\alpha}^{z}-\hat{q}_{\theta}^{z}\right]
$$

where $\hat{\mu}_{t+1, \theta}$ and $\hat{\sigma}_{t+1, \theta}$ are the corresponding one step $\theta$-quantile and scale estimators respectively from the linear conditional quantile process and; $\hat{q}_{\alpha}^{z}$ and $\hat{q}_{\theta}^{z}$ are obtained by inverting the overall distribution of the iid errors. Note that the overall distribution of the iid errors is obtained by splicing GPD with empirical bulk distribution at the threshold as outlined in [11] to get

$$
\hat{F}(z)=1-\frac{m}{N}\left(1+\frac{\hat{\lambda}(z-u)}{\hat{\beta}}\right)^{\frac{-1}{\hat{\lambda}}}
$$

for any $\tau \in(0,1) . \hat{\beta}$ and $\hat{\lambda}$ are the estimated parameters of the Generalised Pareto Distribution (GPD) adopted in formulating the estimated overall distribution of the iid errors. $m$ is the number of exceedances above a chosen threshold $u$ from a sample of size $N$. Inverting $\hat{F}(z)$ we get

$$
\hat{q}_{\tau}^{z}=u+\frac{\hat{\beta}}{\hat{\lambda}}\left[\left(\frac{N}{m}(1-\tau)\right)^{-\hat{\lambda}}-1\right]
$$

Using Equation (4) the estimator of the expected shortfall from standardized errors is given by

$$
\widehat{E S}_{\alpha}(Z)=\frac{\hat{q}_{\alpha}^{z}}{1-\hat{\lambda}}+\frac{\hat{\beta}-\hat{\lambda} u}{1-\hat{\lambda}}
$$

where $\hat{q}_{\alpha}^{z}$ is as defined in Equation (12). Similarly, using Equation (4) we have

$$
\begin{aligned}
\widehat{E S}_{\alpha}(X) & =\frac{1}{1-\alpha} \int_{\alpha}^{1}\left(\hat{\mu}_{t+1, \theta}+\hat{\sigma}_{t+1, \theta}\left[\hat{q}_{\tau}^{z}-\hat{q}_{\theta}^{z}\right]\right) \mathrm{d} \tau \\
& =\hat{\mu}_{t+1, \theta}+\hat{\sigma}_{t+1, \theta}\left[\widehat{E S}_{\alpha}(Z)-\hat{q}_{\theta}^{z}\right]
\end{aligned}
$$

where $\widehat{E S}_{\alpha}(Z)$ is as defined in Equation (13).

\section{Weighted Expected Shortfall}

Suppose $\alpha \in(0,1)$, then we define the Weighted Expected Shortfall for a continuous random variable $X$ as:

$$
W E S_{\alpha}(X)=\int_{\alpha}^{1} \Psi_{\tau}\left(\mu_{t, \theta, \tau}\right) \mu_{t, \theta, \tau} \mathrm{d} \tau
$$

where $\Psi_{\tau}\left(\mu_{t, \theta, \tau}\right)$, are dynamic weights (risk spectrum) that vary from one quantile to another in the integrand and $\alpha$ is fixed. $\mu_{t, \theta, \tau}$ is the quantile at level $\tau$.

Assumption 1 The dynamics of the weight function (risk spectrum) are such that for any risks $X, Y ; \alpha \in(0,1)$ and $\alpha \leq \tau \leq 1$

$$
\Psi_{\tau}\left(\mu_{t, \theta, \tau}^{x}\right) \geq \Psi_{\tau}\left(\mu_{t, \theta, \tau}^{x+y}\right) \text { and } \Psi_{\tau}\left(\mu_{t, \theta, \tau}^{y}\right) \geq \Psi_{\tau}\left(\mu_{t, \theta, \tau}^{x+y}\right)
$$

where $\mu_{t, \theta, \tau}^{x}$ is the quantile at level $\tau$ for loss distribution of $X, \mu_{t, \theta, \tau}^{y}$ is the quantile at level $\tau$ for loss distribution of $Y$ and $\mu_{t, \theta, \tau}^{x+y}$ is the quantile at level 
$\tau$ for loss distribution of $Z=X+Y$

To reduce cumbersomeness in notations, we will drop the superscript on the quantile function, $\mu_{t, \theta, \tau}$ and reintroduce it when referring to another loss distribution different from $X$.

Proposition 1 (Weights) $\Psi_{\tau}\left(\mu_{t, \theta, \alpha}\right)$ defined as

$$
\Psi_{\tau}\left(\mu_{t, \theta, \alpha}\right)= \begin{cases}\frac{\exp \left\{-\left(\mu_{t, \theta, \tau}-\mu_{t, \theta, \alpha}\right)\right\}}{\int_{\alpha}^{1} \exp \left\{-\left(\mu_{t, \theta, \tau}-\mu_{t, \theta, \alpha}\right)\right\} \mathrm{d} \tau} & \tau \geq \alpha \\ 0 & \text { otherwise }\end{cases}
$$

is an admissible risk spectrum.

Proof. We need to show that $\Psi_{\tau}\left(\mu_{t, \theta, \alpha}\right)$ satisfies the three conditions in [12] for an admissible risk spectrum in the definition of a spectral risk measure.

1) Expanding Equation (17) we obtain

$$
\begin{aligned}
\Psi_{\tau}\left(\mu_{t, \theta, \tau}\right) & =\frac{\exp \left\{-\left(\mu_{t, \theta}+\sigma_{t, \theta}\left[q_{\tau}^{z}-q_{\theta}^{z}\right]-\left(\mu_{t, \theta}+\sigma_{t, \theta}\left[q_{\alpha}^{z}-q_{\theta}^{z}\right]\right)\right)\right\}}{\int_{\alpha}^{1} \exp \left\{-\left(\mu_{t, \theta}+\sigma_{t, \theta}\left[q_{\tau}^{z}-q_{\theta}^{z}\right]-\left(\mu_{t, \theta}+\sigma_{t, \theta}\left[q_{\alpha}^{z}-q_{\theta}^{z}\right]\right)\right)\right\} \mathrm{d} \tau} \\
& =\frac{\exp \left\{-\left(\sigma_{t, \theta} q_{\tau}^{z}\right)\right\}}{\int_{\alpha}^{1} \exp \left\{-\left(\sigma_{t, \theta} q_{\tau}^{z}\right)\right\} d_{\tau}} \geq 0
\end{aligned}
$$

as a result of non-negativity of the exponential function. Therefore $\Psi_{\tau}\left(\mu_{t, \theta, \tau}\right) \geq 0$.

2) The first derivative of the weight function with respect to $\tau$ is given by

$$
\begin{aligned}
\frac{\partial}{\partial \tau} \Psi_{\tau}\left(\mu_{t, \theta, \tau}\right)= & \frac{\partial}{\partial \tau}\left[\frac{\exp \left\{-\left(\sigma_{t, \theta} q_{\tau}^{z}\right)\right\}}{\left.\int_{\alpha}^{1} \exp \left\{-\left(\sigma_{t, \theta} q_{\tau}^{z}\right)\right\} \mathrm{d} \tau\right]}\right. \\
= & \frac{\left[\int_{\alpha}^{1} \exp \left\{-\left(\sigma_{t, \theta} q_{\tau}^{z}\right)\right\} \mathrm{d} \tau\right]\left[\frac{\partial}{\partial \tau}-\sigma_{t, \theta} q_{\tau}^{z}\right] \exp \left\{-\left(\sigma_{t, \theta} q_{\tau}^{z}\right)\right\}}{\left(\int_{\alpha}^{1} \exp \left\{-\left(\sigma_{t, \theta} q_{\tau}^{z}\right)\right\} \mathrm{d} \tau\right)^{2}} \\
= & \frac{-\sigma_{t, \theta} \exp \left\{-\left(\sigma_{t, \theta} q_{\tau}^{z}\right)\right\}\left[\frac{\partial}{\partial \tau} q_{\tau}^{z}\right]}{\int_{\alpha}^{1} \exp \left\{-\left(\sigma_{t, \theta} q_{\tau}^{z}\right)\right\} \mathrm{d} \tau} \\
= & -\sigma_{t, \theta} \Psi_{\tau}\left(\mu_{t, \theta, \tau}\right)\left[\left(\frac{N}{m}\right)^{-\lambda}(1-\tau)^{-(\lambda+1)}\right] \\
\leq & 0
\end{aligned}
$$

for $\alpha \leq \tau<1$ which confirms that $\Psi_{\tau}\left(\mu_{t, \theta, \tau}\right)$ is monotonically decreasing in the interval.

3) Observe that

$$
\int_{\tau} \Psi_{\tau}\left(\mu_{t, \theta, \tau}\right) \mathrm{d} \tau=\int_{\alpha}^{1} \Psi_{\tau}\left(\mu_{t, \theta, \tau}\right) \mathrm{d} \tau=\frac{\int_{\alpha}^{1} \exp \left\{-\left(\mu_{t, \theta, \tau}-\mu_{t, \theta, \alpha}\right)\right\} \mathrm{d} \tau}{\int_{\alpha}^{1} \exp \left\{-\left(\mu_{t, \theta, \tau}-\mu_{t, \theta, \alpha}\right)\right\} \mathrm{d} \tau}=1
$$


Replacing $\Psi_{\tau}\left(\mu_{t, \theta, \tau}\right)$ and $\mu_{t, \theta, \tau}$ with their respective estimates in Equation (15) we deduce the corresponding WES estimate as:

$$
\begin{aligned}
\widehat{W E S}_{\alpha}(X) & =\int_{\alpha}^{1} \hat{\Psi}_{\tau}\left(\hat{\mu}_{t, \theta, \tau}\right) \hat{\mu}_{t, \theta, \tau} \mathrm{d} \tau \\
& =\int_{\alpha}^{1} \frac{\exp \left\{-\left(\hat{\mu}_{t, \theta, \tau}-\hat{\mu}_{t, \theta, \alpha}\right)\right\}}{\int_{\alpha}^{1} \exp \left\{-\left(\hat{\mu}_{t, \theta, \tau}-\hat{\mu}_{t, \theta, \alpha}\right)\right\} \mathrm{d} \tau} \hat{\mu}_{t, \theta, \tau} \mathrm{d} \tau \\
& =\hat{\mu}_{t, \theta}+\hat{\sigma}_{t, \theta}\left[\int_{\alpha}^{1} \frac{\hat{q}_{\tau}^{z} \exp \left\{-\left(\hat{\sigma}_{t, \theta} \hat{q}_{\tau}^{z}\right)\right\}}{\int_{\alpha}^{1} \exp \left\{-\left(\hat{\sigma}_{t, \theta} \hat{q}_{\tau}^{z}\right)\right\} \mathrm{d} \tau} \mathrm{d} \tau-\hat{q}_{\theta}^{z}\right] \\
& =\hat{\mu}_{t, \theta}+\hat{\sigma}_{t, \theta}\left[\widehat{W E S}_{\alpha}(Z)-\hat{q}_{\theta}^{z}\right]
\end{aligned}
$$

where $\widehat{W E S}_{\alpha}^{z}$ is the estimated weighted expected shortfall of the standardized errors.

Theorem 1 (Consistency of WES estimator) $\widehat{W E S}_{\alpha}-W E S_{\alpha}=o_{p}(1)$

Proof. Observe that $W E S_{\alpha}$ is a continuous function of $\mu_{t, \theta, \tau}$. Since $\hat{\mu}_{t, \theta, \tau} \stackrel{p}{\longrightarrow} \mu_{t, \theta, \tau}$ by theorem 2 in [11] then by continuous mapping theorem in [13] we have the result.

For a discrete ordered sample $X_{1} \leq X_{2} \leq \cdots \leq X_{n}, \widehat{W E S}_{\alpha}^{d}(X)$ is given by

$$
\widehat{W E S}_{\alpha}^{d}(X)=\sum_{i=n \alpha}^{n} \hat{\Psi}_{i}^{d}\left(X_{i}\right) X_{i}
$$

where $X_{n \alpha}$ is as defined in Equation (6) and

$$
\hat{\Psi}_{i}^{d}\left(X_{i}\right)=\frac{\exp -\left(X_{i}-X_{n \alpha}\right)}{\sum_{i=n \alpha}^{n} \exp -\left(X_{i}-X_{n \alpha}\right)}
$$

Note that as indicated earlier if $n \alpha$ is not an integer then appropriate approximations are made.

\section{Properties of the Weighted Expected Shortfall}

In this section we look at the fundamental properties, lemmas and theorems of the loss random variable as well as the weighted expected shortfall.

Lemma 1 For any random variable $X$, there exists a $U[0,1]$ random variable $U_{X}$ such that $X=F_{X}^{-1}\left(U_{X}\right)$

Proof. This has been proved through a distribution transform in [14] [Lemma 2.1].

Lemma 2 For $\alpha \in(0,1)$ and $X$ integrable we have

$$
W E S_{\alpha}(X)=\operatorname{VaR}_{\alpha}(X)+E\left[\Psi_{\tau}(X)\left(X-\operatorname{VaR}_{\alpha}(X)\right)_{+}\right]
$$

where $(X)_{+}$means $X \mid X>0$ and

$$
\Psi_{\tau}(X)= \begin{cases}\frac{\exp -\left(X-\operatorname{VaR}_{\alpha}(x)\right)}{\int_{\operatorname{VaR}_{\alpha}(X)}^{\infty} \exp -\left(X-\operatorname{VaR}_{\alpha}(X)\right) \mathrm{d} X} & X \geq \operatorname{VaR}_{\alpha}(X) \\ 0 & \text { otherwise }\end{cases}
$$


Proof. By definition

$$
\begin{aligned}
W E S_{\alpha}(X) & =\int_{\alpha}^{1} \Psi_{\tau}\left(\mu_{t, \theta, \tau}\right) \mu_{t, \theta, \tau} \mathrm{d} \tau=\int_{\alpha}^{1} \Psi_{\tau}\left(\mu_{t, \theta, \tau}\right) F_{X}^{-1}(\tau) \mathrm{d} \tau \\
& =\int_{\alpha}^{1} \Psi_{\tau}\left(\mu_{t, \theta, \tau}\right)\left[F_{X}^{-1}(\alpha)+F_{X}^{-1}(\tau)-F_{X}^{-1}(\alpha)\right] \mathrm{d} \tau \\
& =F_{X}^{-1}(\alpha) \int_{\alpha}^{1} \Psi_{\tau}\left(\mu_{t, \theta, \tau}\right) \mathrm{d} \tau+\int_{\alpha}^{1} \Psi_{\tau}\left(\mu_{t, \theta, \tau}\right)\left[F_{X}^{-1}(\tau)-F_{X}^{-1}(\alpha)\right] \mathrm{d} \tau \\
& =\operatorname{VaR}_{\alpha}(X)+E\left[\Psi_{\tau}\left(U_{X}\right)\left(F_{X}^{-1}\left(U_{X}\right)-\operatorname{VaR}_{\alpha}(X)\right)_{+}\right] \\
& =\operatorname{VaR}_{\alpha}(X)+E\left[\Psi_{\tau}(X)\left(X-\operatorname{VaR}_{\alpha}(X)\right)_{+}\right]
\end{aligned}
$$

where

$$
\Psi_{\tau}\left(U_{X}\right)= \begin{cases}\frac{\exp -\left(F_{X}^{-1}\left(U_{X}\right)-\operatorname{VaR}_{\alpha}(x)\right)}{\int_{\alpha}^{1} \exp -\left(F_{X}^{-1}\left(U_{X}\right)-\operatorname{VaR}_{\alpha}(x)\right) \mathrm{d} U_{X}} & F_{X}^{-1}\left(U_{X}\right) \geq \operatorname{VaR}_{\alpha}(x) \\ 0 & \text { otherwise }\end{cases}
$$

Lemma 3 For $\alpha \in(0,1)$ and $X$ integrable we have

$$
\begin{aligned}
W_{\alpha}(X)= & E\left[\Psi_{\tau}(X) X I_{X>\operatorname{VaR}_{\alpha}(X)}\right] \\
& +\Psi_{\tau}\left(\operatorname{VaR}_{\alpha}(X)\right) \operatorname{VaR}_{\alpha}(X)\left[\operatorname{Pr}\left(X \leq \operatorname{VaR}_{\alpha}(X)\right)-\alpha\right]
\end{aligned}
$$

Proof. From Lemma 2 we have

$$
\begin{aligned}
W_{\alpha}(X)= & \operatorname{VaR}_{\alpha}(X)+E\left[\Psi_{\tau}(X)\left(X-\operatorname{VaR}_{\alpha}(X)\right) I_{X>\operatorname{VaR}_{\alpha}(X)}\right] \\
= & E\left[\Psi_{\tau}(X) X I_{X>\operatorname{VaR}_{\alpha}(X)}\right]+\operatorname{VaR}_{\alpha}(X)\left\{1-E\left[\Psi_{\tau}(X) I_{X>\operatorname{VaR}_{\alpha}(X)}\right]\right\} \\
= & E\left[\Psi_{\tau}(X) X I_{X>\operatorname{VaR}_{\alpha}(X)}\right] \\
& +\operatorname{VaR}_{\alpha}(X)\left\{1-\left[1-\Psi_{\tau}\left(\operatorname{VaR}_{\alpha}(X)\right) \operatorname{Pr}\left(X=\operatorname{VaR}_{\alpha}(X)\right)\right]\right\} \\
= & E\left[\Psi_{\tau}(X) X I_{X>\operatorname{VaR}_{\alpha}(X)}\right] \\
& +\Psi_{\tau}\left(\operatorname{VaR}_{\alpha}(X)\right) \operatorname{VaR}_{\alpha}(X)\left[\operatorname{Pr}\left(X \leq \operatorname{VaR}_{\alpha}(X)\right)-\alpha\right]
\end{aligned}
$$

Definition 1 Let $X$ be a bounded, integrable random variable. For $\alpha \in(0,1)$ and $x \in \mathbb{R}$ we define the generalized indicator function

$$
I_{X \geq x}^{(\alpha)}= \begin{cases}I_{X>x}, & \text { if } \operatorname{Pr}(X=x)=0 \\ I_{X>x}+\frac{\operatorname{Pr}(X \leq x)-\alpha}{\operatorname{Pr}(X=x)} I_{X=x}, & \text { if } \operatorname{Pr}(X=x) \neq 0\end{cases}
$$

Lemma 4 Let $X$ be a bounded, integrable random variable. For $\alpha \in(0,1)$ and $x \in \mathbb{R}$, the following holds
1) $0 \leq I_{X \geq \operatorname{VaR}_{\alpha}(X)}^{(\alpha)} \leq 1$
2) $E\left[I_{X \geq \operatorname{VaR}_{\alpha}(X)}^{(\alpha)}\right]=1-\alpha$
3) $E\left[\Psi_{\tau}(X) I_{X \geq \operatorname{VaR}_{\alpha}(X)}^{(\alpha)}\right]=1$ 
4) $E\left[\Psi_{\tau}(X) X I_{X \geq \operatorname{VaR}_{\alpha}(X)}^{(\alpha)}\right]=W E S_{\alpha}(X)$

Proof. For proof of 1) and 2) see Lemma 3.5 in [14].

Prove of 3) follows trivially from admissibility of $\Psi_{\tau}(X)$ in Proposition 1.

To prove 4) we note that if $\operatorname{Pr}\left(X=\operatorname{VaR}_{\alpha}(X)\right)=0$, then $\operatorname{Pr}\left(X \leq \operatorname{VaR}_{\alpha}(X)\right)=\alpha$ hence by lemma 3 we have

$$
E\left[\Psi_{\tau}(X) X I_{X \geq \operatorname{VaR}_{\alpha}(X)}^{(\alpha)}\right]=E\left[\Psi_{\tau}(X) X I_{X>\operatorname{VaR}_{\alpha}(X)}\right]=W_{E S}(X)
$$

If $\operatorname{Pr}\left(X=\operatorname{VaR}_{\alpha}(X)\right)>0$ then

$$
\begin{aligned}
E & {\left[\Psi_{\tau}(X) X I_{X \geq \operatorname{VaR}_{\alpha}(X)}^{(\alpha)}\right] } \\
= & E\left[\Psi_{\tau}(X) X I_{X>\operatorname{VaR}_{\alpha}(X)}\right] \\
& +\frac{\operatorname{Pr}\left(X \leq \operatorname{VaR}_{\alpha}(X)\right)-\alpha}{\operatorname{Pr}\left(X=\operatorname{VaR}_{\alpha}(X)\right)} E\left[\Psi_{\tau}(X) X I_{X=\operatorname{VaR}_{\alpha}(X)}\right] \\
= & E\left[\Psi_{\tau}(X) X I_{X>\operatorname{VaR}_{\alpha}(X)}\right] \\
& +\frac{\operatorname{Pr}\left(X \leq \operatorname{VaR}_{\alpha}(X)\right)-\alpha}{\operatorname{Pr}\left(X=\operatorname{VaR}_{\alpha}(X)\right)} \Psi_{\tau}\left(\operatorname{VaR}_{\alpha}(X)\right) \operatorname{VaR}_{\alpha} \operatorname{Pr}\left(X=\operatorname{VaR}_{\alpha}(X)\right) \\
= & E\left[\Psi_{\tau}(X) X I_{X>\operatorname{VaR}_{\alpha}(X)}\right]+\left[\operatorname{Pr}\left(X \leq \operatorname{VaR}_{\alpha}(X)\right)-\alpha\right] \Psi_{\tau}\left(\operatorname{VaR}_{\alpha}(X)\right) \operatorname{VaR}_{\alpha}(X) \\
= & W E S_{\alpha}(X)
\end{aligned}
$$

Theorem 2 (Subadditivity of WES) Given that $\Psi_{\tau}(X)$ satisfies assumption 1 then $W_{E S}(X)$ is subadditive. That is

$$
W_{E S}(X+Y) \leq W E S_{\alpha}(X)+W E S_{\alpha}(Y)
$$

Proof. By lemma 4 4) we have,

$$
\begin{aligned}
& W S_{\alpha}(X)+W E S_{\alpha}(Y)-W E S_{\alpha}(X+Y) \\
& =E\left[\Psi_{\tau}(X) X I_{X \geq \operatorname{VaR}_{\alpha}(X)}^{(\alpha)}\right]+E\left[\Psi_{\tau}(Y) Y I_{Y \geq \operatorname{VaR}_{\alpha}(Y)}^{(\alpha)}\right] \\
& -E\left[\Psi_{\tau}(X+Y)(X+Y) I_{X+Y \geq \operatorname{VaR}_{\alpha}(X+Y)}^{(\alpha)}\right] \\
& =E\left[X\left(\Psi_{\tau}(X) I_{X \geq \operatorname{VaR}_{\alpha}(X)}^{(\alpha)}-\Psi_{\tau}(X+Y) I_{X+Y \geq \operatorname{VaR}_{\alpha}(X+Y)}^{(\alpha)}\right)\right] \\
& +E\left[Y\left(\Psi_{\tau}(Y) I_{Y \geq \operatorname{VaR}_{\alpha}(Y)}^{(\alpha)}-\Psi_{\tau}(X+Y) I_{X+Y \geq \operatorname{VaR}_{\alpha}(X+Y)}^{(\alpha)}\right)\right]
\end{aligned}
$$

Let

$$
M=\left(X-\operatorname{VaR}_{\alpha}(X)\right)\left(\Psi_{\tau}(X) I_{X \geq \operatorname{VaR}_{\alpha}(X)}^{(\alpha)}-\Psi_{\tau}(X+Y) I_{X+Y \geq \operatorname{VaR}_{\alpha}(X+Y)}^{(\alpha)}\right)
$$

We show that $E[M] \geq 0$. Note that $0 \leq I_{X+Y \geq V a R_{\alpha}(X+Y)}^{(\alpha)} \leq 1$ by lemma 41$)$. When $X>\operatorname{VaR}_{\alpha}(X)$ then $M \geq 0$ since the highest value $I_{X+Y \geq \operatorname{VaR}_{\alpha}(X+Y)}^{(\alpha)}$ can attain is 1 at which point $\Psi_{\tau}(X)-\Psi_{\tau}(X+Y) \geq 0$. When $X=\operatorname{VaR}_{\alpha}(X)$, then $M=0$. Finally, if $X<\operatorname{VaR}_{\alpha}(X)$ then $M \geq 0$ since $\Psi_{\tau}(X+Y) I_{X+Y \geq \operatorname{VaR}_{\alpha}(X+Y)}^{(\alpha)} \geq 0$. Therefore 


$$
\begin{aligned}
& E[M] \\
& =E\left[\left(X-\operatorname{VaR}_{\alpha}(X)\right)\left(\Psi_{\tau}(X) I_{X \geq \operatorname{VaR}_{\alpha}(X)}^{(\alpha)}-\Psi_{\tau}(X+Y) I_{X+Y \geq \operatorname{VaR}_{\alpha}(X+Y)}^{(\alpha)}\right)\right] \\
& \geq 0
\end{aligned}
$$

By Lemma 4 3)

$$
\begin{aligned}
& E\left[\operatorname{VaR}_{\alpha}(X)\left(\Psi_{\tau}(X) I_{X \geq V a R_{\alpha}(X)}^{(\alpha)}-\Psi_{\tau}(X+Y) I_{X+Y \geq V a R_{\alpha}(X+Y)}^{(\alpha)}\right)\right] \\
& =0 \quad \text { by proposition } 1
\end{aligned}
$$

and so we obtain

$$
E\left[X\left(\Psi_{\tau}(X) I_{X \geq V a R_{\alpha}(X)}^{(\alpha)}-\Psi_{\tau}(X+Y) I_{X+Y \geq V a R_{\alpha}(X+Y)}^{(\alpha)}\right)\right] \geq 0
$$

Similarly, it can be shown that

$$
E\left[Y\left(\Psi_{\tau}(Y) I_{Y \geq V^{(\alpha)}(Y)}^{(\alpha)}-\Psi_{\tau}(X+Y) I_{X+Y \geq \operatorname{VaR}_{\alpha}(X+Y)}^{(\alpha)}\right)\right] \geq 0
$$

Therefore

$$
W E S_{\alpha}(X)+W E S_{\alpha}(Y)-W E S_{\alpha}(X+Y) \geq 0
$$

Remark Note that both assumption 1 and theorem 2 can be generalized for any number of losses.

Theorem 3 (Monotonicity of WES) For $\alpha \in(0,1)$ WES $_{\alpha}(X)$ is monotonic. That is if $X \leq Y$ always then

$$
W S_{\alpha}(X) \leq W E S_{\alpha}(Y)
$$

Proof. The result follows from Proposition 3.5(i) in [15] and monotonicity of both the quantile function and the Lebesgue integral.

Theorem 4 (Coherence of WES) Given that $\Psi_{\tau}(X)$ satisfies assumption 1 then $W_{E S}(X)$ is coherent.

Proof. We show that $W E S_{\alpha}(X)$ satisfies the four axioms of definition 2.7 in [4]. We note that the proves of axiom 1) and 3) are trivial. Axiom 2) and 4) follows from theorems 2 and 3 respectively.

\section{Application to Risk Measurement for NSE 20 Share Index}

We compare risk estimates from VaR, ES and WES using NSE 20 Share index data from January 2008 to March 2021. Returns and loss returns are calculated from the price data using Equations (1) and (2) respectively.

Table 1 reports summary statistics of the data which shows that the data is heavy tailed and skewed to the left. Moreso, the reported p-value from the ADF test implies that the data is stationary at $5 \%$ significance level.

From Figure 1 we observe some level of volatility clustering which is common in most financial data sets.

${ }^{3}$ Nairobi Stock Exchange. 
Table 1. Sample statistics.

\begin{tabular}{cc}
\hline \multicolumn{2}{c}{ Statistical properties of the loss return data } \\
\hline minimum & -0.09018 \\
maximum & 0.08243 \\
median $\left(Q_{1}, Q_{3}\right)$ & $0.00013(-0.00374,0.00406)$ \\
mean \pm sd & $0.00017 \pm 0.00843$ \\
skewness & -0.37124 \\
kurtosis & 16.04896 \\
ADF test p-value & 0.01 \\
\hline
\end{tabular}

$Q_{1}$, Lower quartile $Q_{3}$, Upper quartile sd, standard deviation.

NSE 20 share index loss return

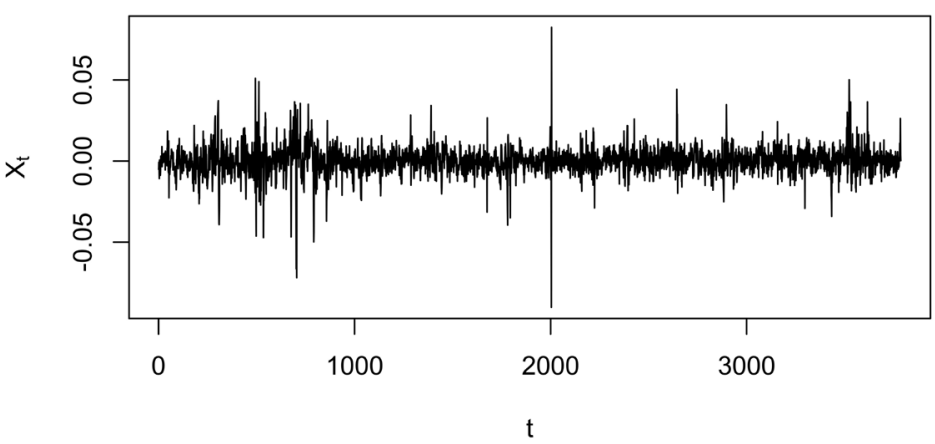

Figure 1. NSE 20 share, loss return time series.

The ACF and PACF plots of the data in Figure 2 suggests autocorrelation in the series of up to order two which informs the number of lags in our QAR process. Therefore using extreme quantile autoregression we obtain the following model for the loss return

$$
\begin{aligned}
X_{t}= & -0.00008188+0.2932 X_{t-1}+0.1330 X_{t-2} \\
& +\left(0.002647+0.1749\left|\varepsilon_{t-1}\right|+0.06753\left|\varepsilon_{t-2}\right|\right) Z_{t}
\end{aligned}
$$

where $Z_{t}$ follows the extreme value distribution given by Equation (11).

Based on p-values in Table 2 all the the estimated parameters of the quantile process are significant at 5\% significance level except the constant of the central quantile process.

The ACF plot in Figure 3 confirms independence of the resulting standardized errors. Hence using the threshold $u=2.038538$, to ensure that $10 \%$ of the errors are classified as extreme, we obtain the following estimates of the shape and scale parameters from the GPD fit.

As outlined in Table 3, the $\mathrm{p}$-values of all the parameter estimates are less than $5 \%$ significance level implying that the distribution fits the data well. Figure 4 shows a plot of the corresponding risk estimates at $97.5 \%$ confidence level.

From Figure 4 we observe that risk estimates from ES are slightly higher than those from WES confirming that WES is less conservative compared to 
NSE 20 ACF

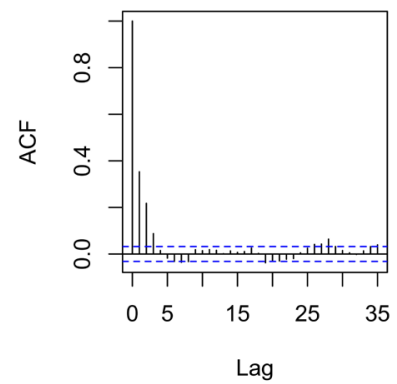

NSE 20 PACF

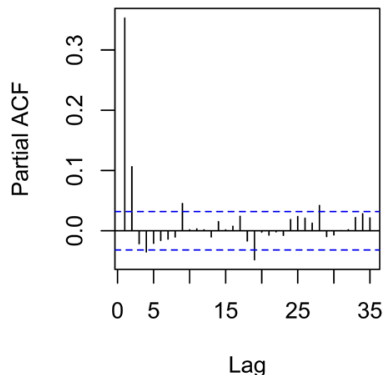

Figure 2. Autocorrelation and Partial Autocorrelation Plot of the loss return series.

Series 1

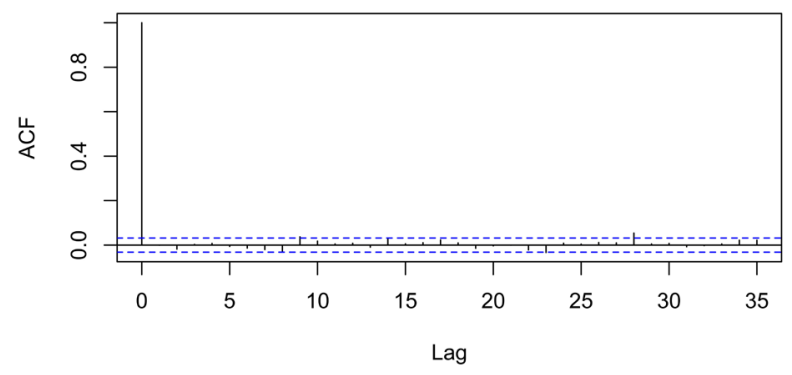

Figure 3. Autocorrelation Plot of the standardized errors.

Risk estimates at $\mathbf{9 7 . 5 \%}$ confidence level

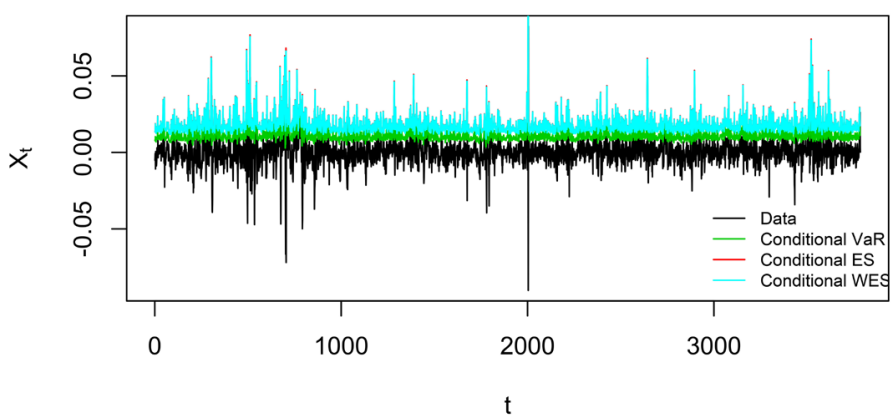

Figure 4. Data plot superimposed with risk estimates from considered Risk measures.

Table 2. Parameter estimates of the central quantile and the scale.

\begin{tabular}{cccccc}
\hline & Term & Estimate & Std. Error & t-value & p-value \\
\hline \multirow{3}{*}{ Central } & constant & 0.00008187868 & 0.0001184322 & 0.6913549 & 0.24471 \\
& QAR(1) & 0.2931358 & 0.0251979805 & 11.6333058 & 0.0000 \\
& QAR(2) & 0.1329694 & 0.0227462397 & 5.8457766 & 0.0000 \\
\hline \multirow{3}{*}{ Scale } & constant & 0.002646745 & 0.0001448444 & 18.273030 & 0.0000 \\
& QAR(1) & 0.174948799 & 0.0273538824 & 6.395758 & 0.0000 \\
& QAR(2) & 0.067533867 & 0.0231822250 & 2.913175 & 0.0018 \\
\hline
\end{tabular}


Table 3. Parameter estimates of the Central quantile and the scale.

\begin{tabular}{ccccc}
\hline Parameter & Estimate & Std. Error & t-value & p-value \\
\hline Shape & 0.2445236 & 0.06464320 & 3.7826546 & 0.0000 \\
Scale & 0.9666750 & 0.07864687 & 12.2913347 & 0.0000 \\
\hline
\end{tabular}

ES. However, risk estimates from both ES and WES are higher than those from VaR because VaR failed to appreciate severity of losses beyond the $97.5 \%$ confidence threshold.

\section{Conclusion and Recommendations}

We have improved the adjusted extreme conditional quantile estimator in [11] and used it to obtain the one-step-ahead risk estimators for Var, ES and WES. The three estimators were then used to quantify risk in the NSE 20 share index portfolio. Consistency and coherence of the proposed Weighted expected shortfall were also proved. It was observed that the WES is less conservative compared to ES.

\section{Data Availability}

The authors used NSE 20 share index data obtained from the Nairobi Stock Exchange (NSE).

\section{Acknowledgements}

The authors thank the Pan-African University of Basic Sciences, Technology and Innovation (PAUSTI) for funding this research.

\section{Conflicts of Interest}

The authors declare no conflicts of interest regarding the publication of this paper.

\section{References}

[1] Artzner, P., Delbaen, F., Eber, J.-M. and Heath, D. (1999) Coherent Measures of Risk. Journal of Mathematical Finance, 9, 203-228. https://doi.org/10.1111/1467-9965.00068

[2] Acerbi, C., Nordio, C. and Sirtori, C. (2001) Expected Shortfall as a Tool for Financial Risk Management. arXiv Preprint cond-mat/0102304.

[3] Acerbi, C. and Tasche, D. (2002) On the Coherence of Expected Shortfall. Journal of Banking \& Finance, 26, 1487-1503. https://doi.org/10.1016/S0378-4266(02)00283-2

[4] Roccioletti, S. (2015) Backtesting Value at Risk and Expected Shortfall. Springer. https://doi.org/10.1007/978-3-658-11908-9

[5] Basel Committee on Banking Supervision (2013) Consultative Document-Fundamental Review of the Trading Book: A Revised Market Risk Framework (tech. rep.). https://www.bis.org/publ/bcbs265.pdf

[6] Gneiting, T. (2011) Making and Evaluating Point Forecasts. Journal of the American Statistical Association, 106, 746-762. https://doi.org/10.1198/jasa.2011.r10138 
[7] Acerbi, C. and Szekely, B. (2014) Back-Testing Expected Shortfall. Risk, 27, 76-81.

[8] Cont, R., Deguest, R. and Scandolo, G. (2010) Robustness and Sensitivity Analysis of Risk Measurement Procedures. Quantitative Finance, 10, 593-606. https://doi.org/10.1080/14697681003685597

[9] Peracchi, F. and Tanase, A.V. (2008) On Estimating the Conditional Expected Shortfall. Applied Stochastic Models in Business and Industry, 24, 471-493. https://doi.org/10.1002/asmb.729

[10] Leorato, S., Peracchi, F. and Tanase, A.V. (2012) Asymptotically Efficient Estimation of the Conditional Expected Shortfall. Computational Statistics \& Data Analysis, 56, 768-784. https://doi.org/10.1016/j.csda.2011.02.020

[11] Kithinji, M.M., Mwita, P.N. and Kube, A.O. (2021) Adjusted Extreme Conditional Quantile Autoregression with Application to Risk Measurement. Journal of Probability and Statistics, 2021, Article ID: 6697120. https://doi.org/10.1155/2021/6697120

[12] Acerbi, C. (2002) Spectral Measures of Risk: A Coherent Representation of Subjective Risk Aversion. Journal of Banking \& Finance, 26, 1505-1518. https://doi.org/10.1016/S0378-4266(02)00281-9

[13] Whitt, W. (1980) Some Useful Functions for Functional Limit Theorems. Mathematics of Operations Research, 5, 67-85. https://doi.org/10.1287/moor.5.1.67

[14] Embrechts, P. and Wang, R. (2015) Seven Proofs for the Subadditivity of Expected Shortfall. Dependence Modeling, 3, 126-140. https://doi.org/10.1515/demo-2015-0009

[15] Brutti, R.M. (2018) A Theory for Combinations of Risk Measures. arXiv e-prints, arXiv-1807. 